\title{
Patient Condition Monitoring Modular Hospital Robot
}

\author{
K. A. Mamun ${ }^{1}$, A. Sharma1 , F. R. Islam ${ }^{1}$, A. S. M. Hoque ${ }^{2}$, T. Szecsi ${ }^{3}$ \\ ${ }^{1}$ School of Engineering and Physics, The University of the South Pacific, Suva, Fiji. \\ 2 Department of Industrial and Production Engineering Jessore University of Science and Technology, \\ Jessore-7408, Bangladesh. \\ ${ }^{3}$ School of Mechanical and Manufacturing Engineering Dublin City University, Dublin 9, Ireland. \\ * Corresponding author. Tel.: +679 323 31760; email: mamun_k@usp.ac.fj \\ Manuscript submitted January 13, 2016; accepted March 20, 2016. \\ doi: $10.17706 /$ jsw.11.8.768-786
}

\begin{abstract}
In this paper an intelligent system for remote patient physical condition monitoring service module for an Intelligent Robot Swarm for Attendance, Recognition, Cleaning and Delivery (iWARD) [1] is reported. The system algorithm and module software is implemented in $\mathrm{C} / \mathrm{C}++$, and the Orca robotics [2] components use the OpenCV [3] image analysis and processing library. The system is successfully tested on Linux (Ubuntu) platform as well as on a web server. The patient condition monitoring system can remotely measure the following body conditions: body temperature (BTemp), heart rate (HR), electrocardiogram (ECG), respiration rate (RR), body acceleration (BA) using sensors attached to the patient's body. The system also includes an RGB video camera and a 3D laser sensor, which monitor the environment in order to find any patient lying on the floor. The system deals with various image-processing and sensor fusion techniques. The iWARD patient condition monitoring module evaluation tests were carried out in front of thirty healthcare professionals (doctors, nurses, nursing lecturers and healthcare assistances etc) during the final review meeting of the consortium and in two teaching hospitals (in Newcastle and San Sebastian, 2009) in Europe. The post iWARD system improved upon the prototype by adding a 3D laser sensor and replacing the original camera with a high quality Pan-Tilt-Zoom (PTZ) camera and implementing the identity detection methods. This allowed for the use of more robust patient condition monitoring algorithms. The outcomes of this research have significant contribution to the robotics application area in the hospital environment.
\end{abstract}

Key words: Intelligent system, patient condition monitoring, hospital robot.

\section{Introduction}

The shortage of healthcare staff has been a big issue over the past decade in the developed world. Hospitals are overcrowded with patients, with stressed out workers and it leads to errors occurring, patients waiting too long for assistance and a lack of hygiene throughout the hospitals. According to the Irish Health Service Executive, over the past few years cleaning costs for Irish hospitals have risen sharply from €99.7 million to €157.2 million but only 9\% of hospitals received good rating for cleaning [4]. As time progresses, this problem would not alleviate rapidly but rather increase the problems that are already occurring. So, using robots in hospital environments for assisting nurses in their everyday tasks is not uncommon in the developed world. Till now the major tasks of hospital robot systems like cleaning, surveillance, guidance, delivery and patient physical condition monitoring, etc. have been absent in one individual robot platform. 
The iWARD [1] robot is such this type of robot where all major tasks could be achieved. Patient physical condition monitoring is a task for a robot which is a critical component of medical care. Vital signs of a patient that need to be measured include body temperature (BTemp), heart rate (HR), electrocardiogram (ECG), respiration rate (RR), body acceleration (BA) need to be processed and analysed to make a decision regarding the patient's health condition. In a hospital environment sometimes a patient may have fallen on the floor either consciously or unconsciously. Within this scenario, the robot, while patrolling the hospital, analyzes the sensors' data and attempts to detect a person on the floor. If the robot finds such a person it raises an event that is analysed by the robot swarm system. The nurses' station is also informed in order to take action. Since it is highly unlikely that during daytime the robot can detect a fallen person before he/she is given help, this type of detection is only required during night time when there is only a limited presence of staff in corridors and other areas.

In this research the robot can remotely collect information about the physical condition of patients. The data is stored in the shared knowledge of the robot system as well as web server and can be used by staff members, other registered doctors of other hospitals through the internet. If a patient's condition is critical or lying on the floor the robot raises an event and informs the robot system and the nurses' station. The robot is equipped with a small light alarming system to alarm for the hospital staff. This scenario can be performed either routinely, while the robot is patrolling, for each patient in the vicinity of the robot, or the robot can be ordered to take measurements from a specific patient.

\section{Literature Survey}

With the development of robotics technologies, the application of robots have been extended more and more widely, i.e. in manufacturing cars, industrial assembly lines, health-care, and so on. The impact of robotics applications in healthcare could be huge in terms of health, societal and economic benefits. Without compromising the quality of care, robotics applications could offer the promise of sustainable and affordable health care provision. Nowadays different types of robots for different applications are available commercially and some of them are used as security and delivery robot for hospital environment separately. The Care-o-bot [5] and Skillgent [6] are such type of robot which capable of monitoring patient physical condition remotely, although none of them have the standalone modular feature. They are designed with built in features. On the other hand, there are a large number of systems to monitor patient physical condition (i.e. vital signs) available commercially. They are not integrated with autonomous mobile platforms as well as with a web server. To date, researchers have developed numerous approaches to collaborative robot control to solve different application problems. Cao et al. [7] deserve credit for attempting the categorization of different architectures in the cooperative, multi-robot domain. There has been a decade and a half of research aimed at providing robots with a behavioral or task-based decomposition of the control system since Brooks [8] introduced it as an alternative to the traditional functional approach. The application of behavior-based robotics to groups of robots has been explored extensively in the latter half of the 1990s (e.g. collective autonomous agents [9]) and architectures for cooperative control have recently been introduced [10]. Much work is focused on how different behaviors have to be combined to achieve a desired effect that solves a given problem. Proposed solutions include hard-coded or learned weights [11]. Attempts have also been made to analytically determine the minimum information requirements for solving a task [12] and for automatic generation of robot teams [13]. However, most of these systems have not tackled the problems of distributed collaborative behaviors and distribution of resources across robots.

Currently, self-navigating mobile robots have been used in many hospital/medical applications: transport in the X-ray region, chemical gas detection system of toxic residues or hazard points, remote inspection for 
detection of high temperature and radiation, aided-radiotherapy [14-16]. Shieh proposed a design concept of an intelligent hospital service robot (IHSR) aimed at saving human resources and also improving the hospital services [17]. Takahashi [18] has been developing the MKR (Muratec Keio Robot), an autonomous Omni-directional mobile transfer robot system for hospital environment to support a delivery system. Rafflin and Fournier [19] presented FIRST, a Friendly Interactive Robot for Service Tasks, designed to carry heavy loads in hospitals. The uBOT-5 [20] is the result of project ASSIST, which can quietly follow its owner around the house, take care of the cleaning, give reminders about medication, help with shopping and make communication with doctors. The uBOT-5 also has the ability to recognise when its owner has fallen or become unresponsive, allowing it to immediately dial 911 for medical attention and relay important information to caregivers en route [21]. This robot is designed to work in home environment, instead of in a hospital environment.

To improve the quality of healthcare, these focal issues emerge: fast identification and location of patients needing immediate attention; reduction of human errors; effective cleaning in hospitals; wider reach of specialist medics, possibly attending patients remotely. To achieve this, iWARD presents a robot swarm delivering support to oversee activities in healthcare environments, providing a multipurpose, cost-effective and scalable solution to enhance quality of healthcare. The robots can be equipped with different adaptable hardware components for remote patient physical condition monitoring, floor cleaning and delivery of food, linen, medicine etc. All mobile robots are capable of providing patients and visitors with guidance and information. It provides easy to use but high tech interaction interfaces like voice control through mobile and fix-mounted robots. The swarm based approach unburdens the nursing staff from the details of robot control and central coordination reducing the complexity of robot control to that of a chat, having the swarm negotiating which robot to be used for each job, shortening the reaction time, reducing human error and increasing efficiency to provide better patient care. Modular robotics applications have very limited uses in hospital environments. The selected module sensors (mounted on the autonomous robot platform) provide dynamic data for all the proposed modules (i.e. patient physical condition monitoring) in this research, as opposed to approaches using stationary sensors.

\section{Module Hardware Design}

The iWARD philosophy suggests that instead of large, specialized robots, general-purpose, small robots with attached service modules are to be used which provides easy configuration, easy expansion, low cost and easy optimization of task schedules. According to these principles, service modules are attached to a mobile, self-navigating robot platform (Fig. 1); these modules are used to execute various scenarios in the hospital. Service modules should allow for interchangeability, Plug-and-Play, and have self-contained sensors, computing devices and other hardware and software, powersave mode and are developed as Orca robotics components. One of the major design features of the iWARD robot swarm is that there is no central computer in the system. Instead, the robots are equipped with a level of intelligence to negotiate and schedule tasks between themselves dynamically - the robot swarm is a self-organizing system. The robots themselves decide which of them is most suitable to perform a given task at a given time. The interchangeable robot modules can be attached to the robot bases according to demand. Artificial intelligence learning mechanisms support many robot functions. These functional modules would operate while attached to a robot base in a hospital environment, patrolling the hallways and patient wards for human movement and report any unusual activity defined as the modules tasks to a central terminal and to the swarm system. Sensor fusion and image processing through a cost effective approach are two primary features of this research. The features of the final product of this research also include patient lying on the floor detection and patient physical condition monitoring. The following camera and sensors have been 
used for this module: TV7240 [22] is a low-cost pan-and-tilt IP camera; 03D100 PMD 3D laser sensor from Ifm Electronics [23] is the first industrial 3D sensor that can detect objects in three dimensions at a glance and EQ-01 Sensor Unit [24] consists of the EQ-01 Monitoring Belt and EQ-01 Sensor Electronics Module (SEM).

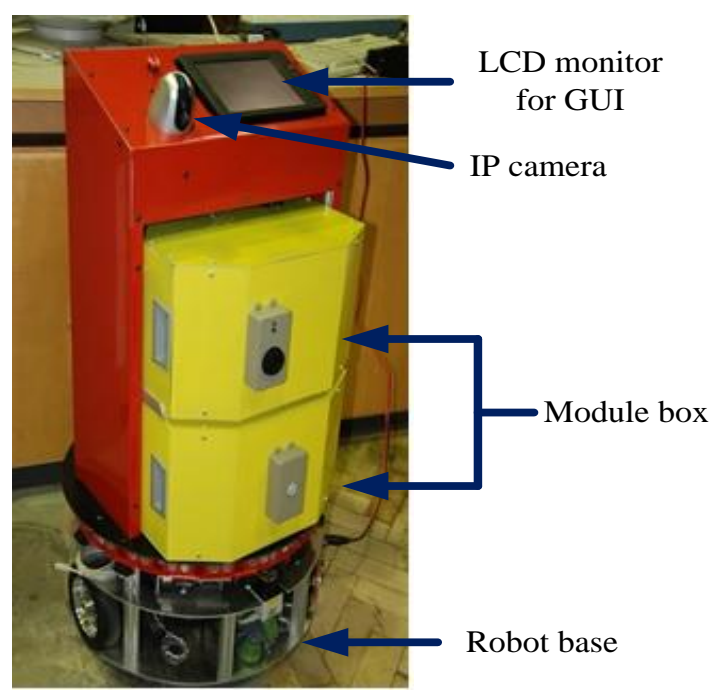

Fig. 1. Self-navigating robot platform with service modules.

\section{Module software Development}

\subsection{Patient Lying on the Floor}

Detecting any patient who has fallen and is lying on the floor consists of face a detector, badge detector, colour segmentation, image fusion, background segmentation, blob creator, and decision making based on Artificial Neural Networks (ANN). Fig. 2 shows the flow diagram of the detection of a patient lying on the floor scenario module software algorithm.

The PTZ video camera and the PMD 3D laser sensor are hardware shared by both scenarios: intruder monitoring and patient on the floor detection. The steps of detecting a patient lying on the floor are as follows:

1) The mission scheduler component starts the Situation Recognition module (patient lying on the floor).

2) The PIR sensor detects motion in the vicinity of the robot. This triggers the activation of all sensors (including the video camera and laser sensor).

3) The module obtains the location ID through the Orca interface.

4) An image is grabbed from the IP camera, and the face detector searches for a positive match. If no face is found the Pan/Tilt/Zoom mechanism of the camera is used to get a new image frame in the vicinity of the robot platform by changing the camera orientation and field of view. If a face is found, its size is measured. An upper body area is defined proportioned relative to the size of the face to select a region of interest.

5) At the same time the PMD 3D laser sensor is activated to provide distance information for each pixel of the colour image. Pure coloured images make image recognition difficult because the pixels do not contain depth information. In this case colour and texture gradient techniques can be applied to separate foreground and background objects. This, however, is error-prone, especially in varying lighting conditions. In order to improve the robustness of the imaging software, imaging sensor fusion can be applied. In this research, the camera image is combined with the image from the 3D laser sensor, 
which gives depth information to each coloured pixel in the camera image. Fig. 3 shows an image from the video camera (left) and the laser image of the same scene (right); the laser distance data was converted into greyscale intensity image (the darker the pixel, the further away it is from the sensor). In order to be able to match the pixels in both images, they first need to be rectified by compensating for distortions (lens distortions, perspective distortions). The depth information of coloured pixels can then be used to set depth thresholds relative to found faces and badges, thus making background subtraction easier.

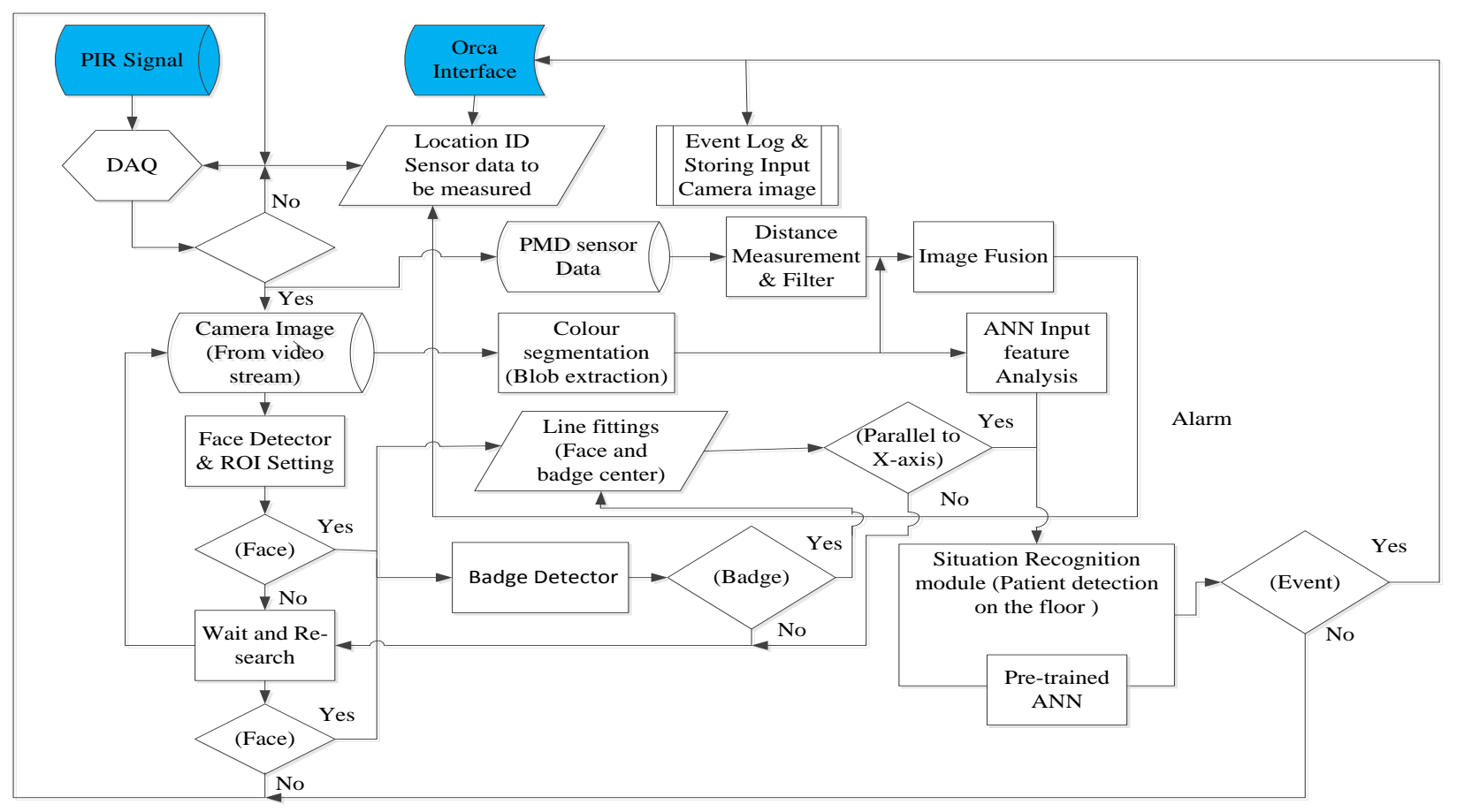

Fig. 2. Flow diagram of the detection of patient lying on the module software algorithm.

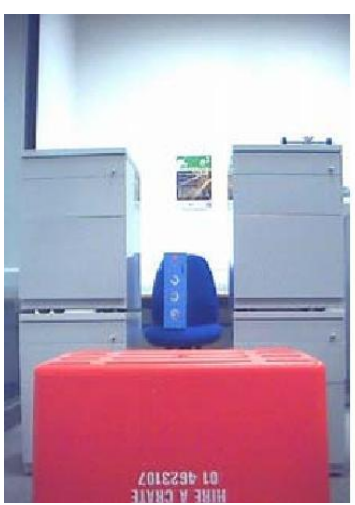

Fig. 3. Camera and laser image.

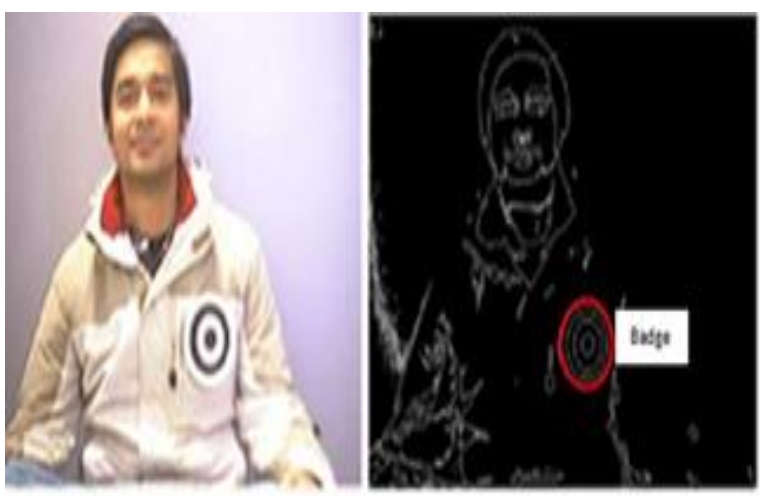

Fig. 4. Badge detection.

6) It is assumed that all authorised persons in the hospital are wearing identification badges. It consists of at least 5 black and white concentric circles (i.e. badge). The programme searches the upper body to find this badge. Badge detection in this research is based on finding all contours in an image and creating bounding ellipses around them. In the next step, the centre of each ellipse is calculated and the corresponding contours are grouped together if they are reasonably close to each other (Fig. 4). Since the contours related to the badge all have concentric circles, the centres of their bounding ellipses will 
remain concentric regardless of the viewing angle. Once a badge is located in the image, its position relative to ground level (similarly to face detection) can be a clue that the person wearing the badge is lying on the floor. If both a face and badge are detected, their relative size and orientation can be used to decide if they belong to the same person and if the person is lying on the floor.

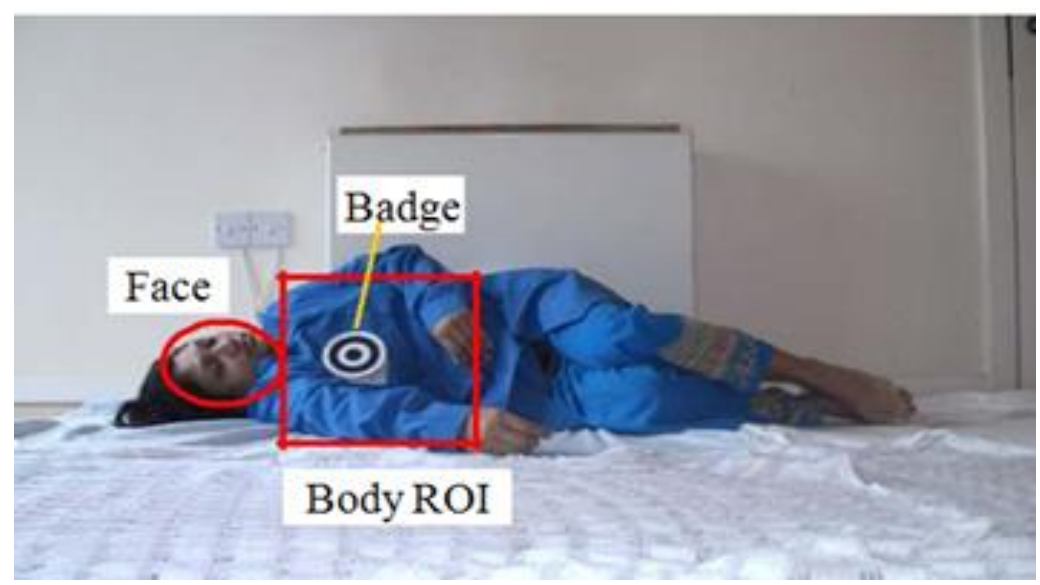

Fig. 5. Camera and laser image.

Relative to the face and in proportion with its size, a rectangle representing the size of the upper body is drawn in the direction towards the badge. This rectangle becomes the region-of-interest (ROI) in which the badge must be located in order to belong to the same person. The relative position of the badge and face in Fig. 5 indicates that the badge belongs to the person and the person is lying on the floor.

7) If a face is found in the current frame, an upper body area is defined proportioned relative to the size and position of the face. A region of interest (ROI) is marked on the upper body (Fig. 5). Then the badge detector searches for a badge within the ROI.

8) If the badge is in the ROI and the ROI is either on the left or right of the face then it is one of the indications to assume that a patient is lying on the floor. This step also draws a line between the face centre and badge centre (if any) to confirm the orientation of the patient's body. The angle of the line $\theta$ defines the orientation of the body relative to the vertical direction. A near- $90^{\circ}$ angle (within a certain tolerance range of $\pm \alpha$ ) is an indication that the patient is lying horizontally (Fig. 6). The position of the line relative to ground level (D) confirms if the patient is lying on the floor. ' $\mathrm{D}$ ' is calculated based on the current zoom level of the camera and the size of the face in the current frame.

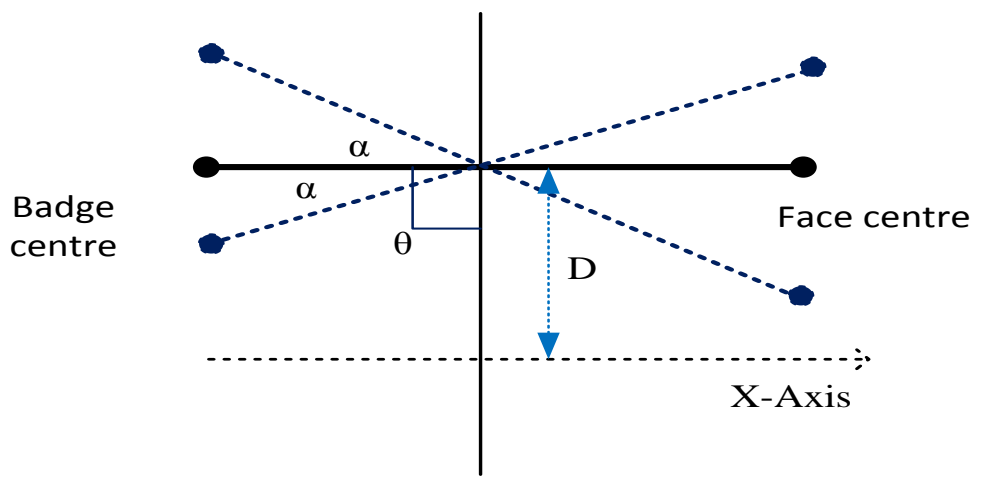

Fig. 6. Face and badge centre line orientation relative to the $x$-axis of the image frame.

9) If only either a face or a badge is found in the frame, its relative position to ground level is again an 
indication that a patient may be lying on the floor. Fig. 7 sows an illustration of face detection relative to ground level.

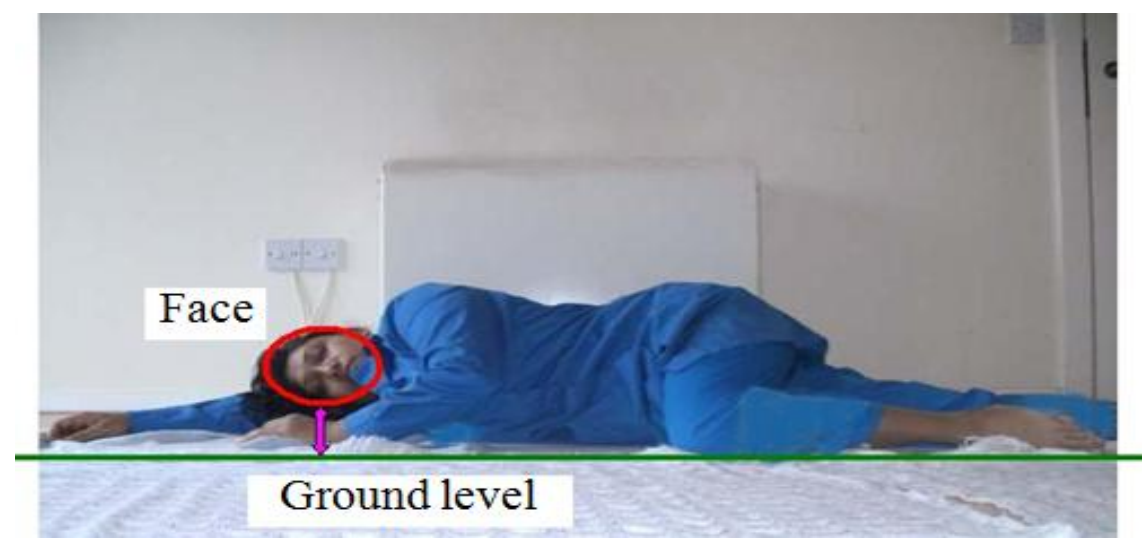

Fig. 7. Face detection relative to ground level.

10) In some cases a face and/or badge might be occluded from the view of the camera, and as such only a segmented dress colour blob will be combined with the PMD sensor data. If neither a face nor a badge is found in the frame, other imaging techniques are required. For this, the software attempts to find colour blobs representing human skin and clothing in the frame. The colour segmentation component (using the HSV colour space) defines an eventual human body based on a patient's unique dress colour (i.e. blue) and skin colour (Fig. 8 (a, b)). Once all skin and clothing blobs are found, using the fused sensor data (colour and distance) the average distance of each blob is defined, and blobs with similar distances are grouped together (Fig. 8 (c)). A combined blob with correct size (depending on the current zoom value and the size of the blob in the frame) is considered to be a possible image of a person in the frame (Fig. 9).
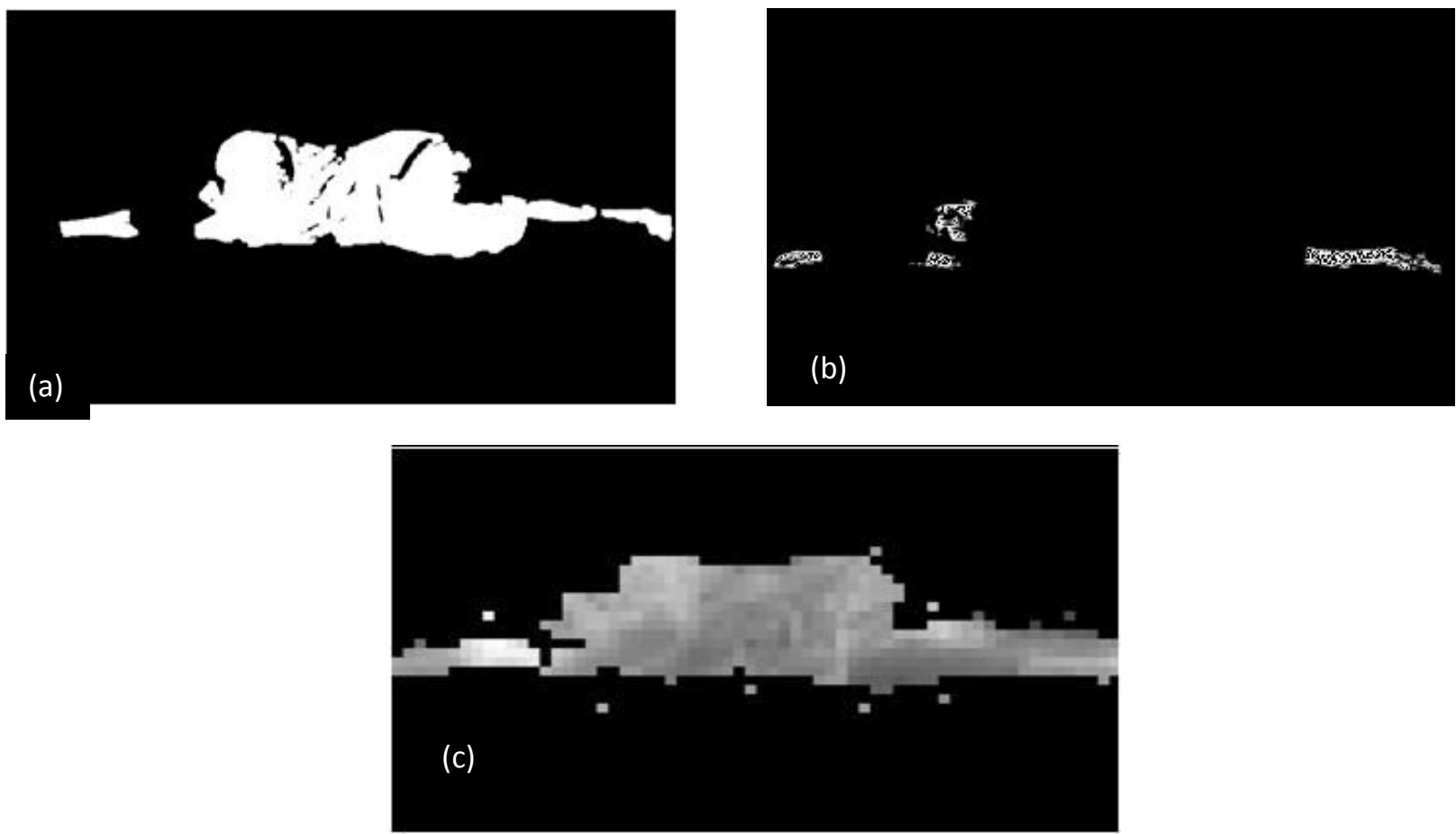

Fig. 8. (a) Binary image of separated human clothing, (b) Binary image of separated human skin, (c) Laser distance image of human body. 


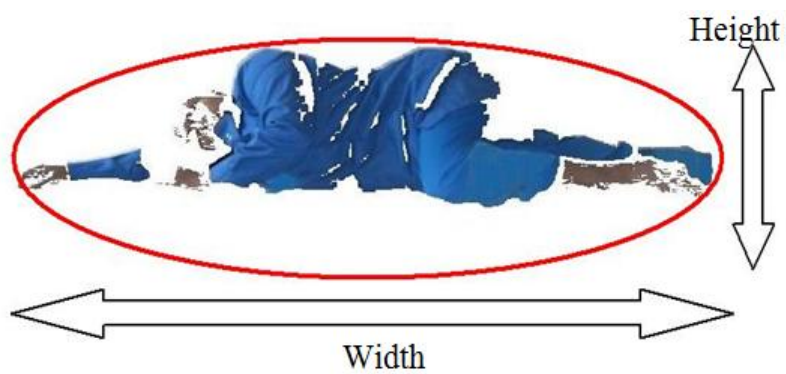

Fig. 9. Combined blobs of human skin and clothing.

11) To eliminate potential errors, the fused sensor data is used to separate background pixels that are clearly not part of the foreground object. An envelope is drawn around the foreground object (its size corresponds to the size of an average human), and all pixels that are outside this envelope are removed from the image. However, the truncated image still contains pixels that cannot be part of the foreground object. This is because the laser distance sensor cannot distinguish pixels that belong to the floor and pixels of objects that lie directly on the floor. By repeating the blob search technique on the truncated image, those pixels that are too far away in the HSV colour scheme from the skin and clothing colours are eliminated (Fig. 9).

12) The resulting combined image is then analysed to retrieve the parameters (i.e. Free-man chain code, differential freeman chain code, convexity defects and hue-saturation histogram) required for the ANN to obtain a classification of the detected object. Chain codes are the most size-efficient representations of rasterised binary shapes and contours. Chain code compression has become a propulsive research subject over the last decade [25 - 29]. The methods presented to-date has been primarily based on the Huffman coding of individual chain code (FCC) symbols [30]. In this research various characteristics of a contour are used to decide whether it may belong to a person lying on the floor. To extract the contour of foreground objects, the sensor fusion and colour separation techniques described above are used.
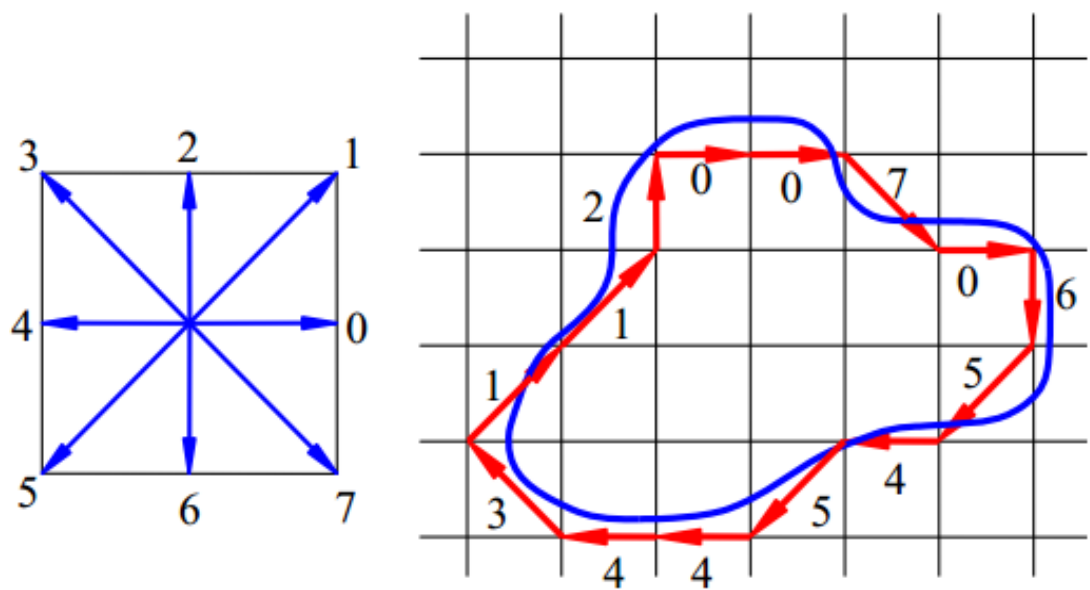

Fig. 10. Freeman chain-code of a contour.

The first contour characteristic is its Freeman chain-code (FCC). It describes a contour as a sequence of vectors representing elementary movements in any of eight directions from one pixel of the image to another. The vectors are defined so that the resulting movements are the closest to the original contour (Fig. 10). Thus, the FCC of the contour is (starting at the leftmost point): 1-1-2-0-0-7-0-6-5-4-5-4-4-3. 
It would be difficult to apply the chain-code directly to characterise the contour because even slight variations of the contour would result in a completely different code. Instead, in this research the distribution (histogram) of the eight moves is used. Although the histogram of the FCC gives a good overall description of the contour, it may fail to distinguish between significantly different shapes. For example, the three different shapes in Fig. 11 will all result in the same FCC histogram (shown in Fig. 12) as long as their overall height and width are identical.
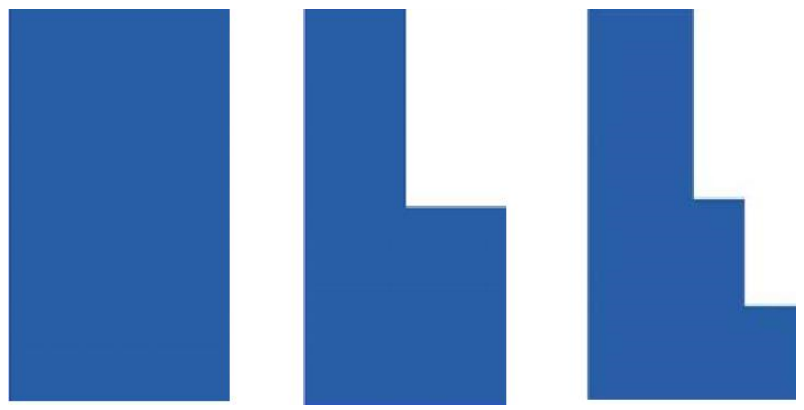

Fig. 11. Shapes for Freeman chain-code.

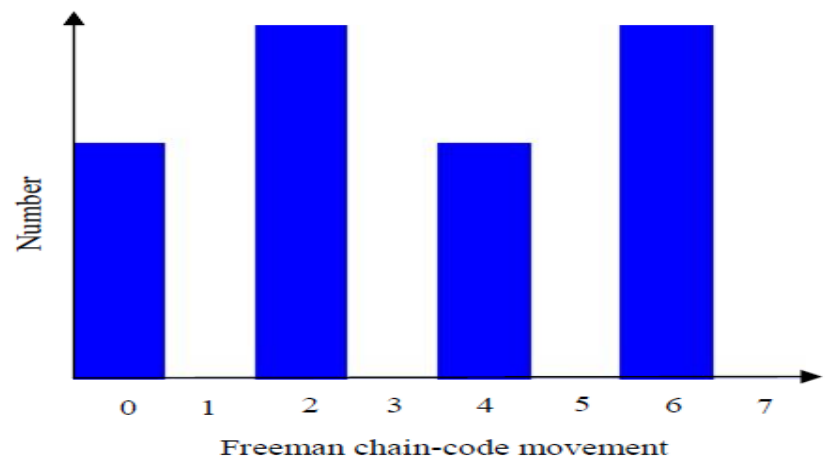

Fig. 12. Freeman chain-code histogram of the three contours shown in Fig. 11.

In order to eliminate the above problem, a new chain code called Differential chain-code (DCC) is suggested in this research. It is based on the FCC, but instead of marking the actual direction of the movements, only the changes in direction are marked (Table 1).

Table 1. Differential Chain Codes

\begin{tabular}{ll}
\hline DCC code & \multicolumn{1}{c}{ Meaning } \\
\hline L45 & Left turn by 450 \\
L90 & Left turn by 900 \\
L135 & Left turn by 1350 \\
R45 & Right turn by 450 \\
R90 & Right turn by 900 \\
R135 & Right turn by 1350 \\
S & No change in direction (same direction as previous)
\end{tabular}

The motions corresponding to the DCC codes are shown in Fig. 13. Given that the endpoints of an 
elementary movement have to coincide with the pixels of the image, no other movements are possible. Using this code, the DCC of the contour in Fig. 10 is: R90-S-L45-R90-S-R45-L45-R90-R45-R45-L45-L45-R45-S-R45.

One of the advantages of the new DCC over FCC is that the DCC histogram does not depend on the orientation of the object. However this differential chain-code (DCC) is able to detect the difference between the shapes shown in Fig. 11. The DCC histograms of these three shapes are shown in Fig. 14.

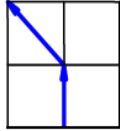

L45

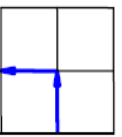

L90

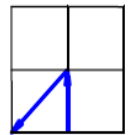

L135

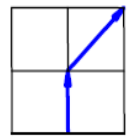

R45

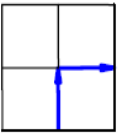

R90

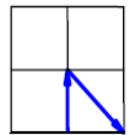

R135

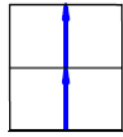

$\mathrm{S}$

Fig. 13. Differential chain-code movements.

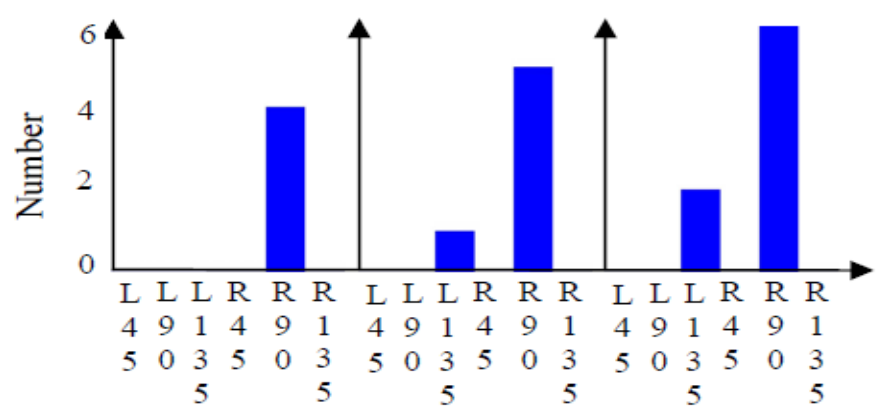

Differential chain-code

Fig. 14. Differential chain-code histogram.

The next major parameter of a contour used in this research is its convexity defects [32]. A convexity defect is an area where the contour deviates from a convex curve. The contour of any shape cannot be described by a convex curve, so the contour has convexity defects. The areas that lie between the convex hull and the contour are the convexity defects (Fig. 15). The easiest way to characterise each defect is to draw a triangle using the points where the defect starts ( $a$ and b) and the deepest point of the defect (c). Since the actual shape of the defect is not of great importance, it is sufficient to calculate its depth (D).

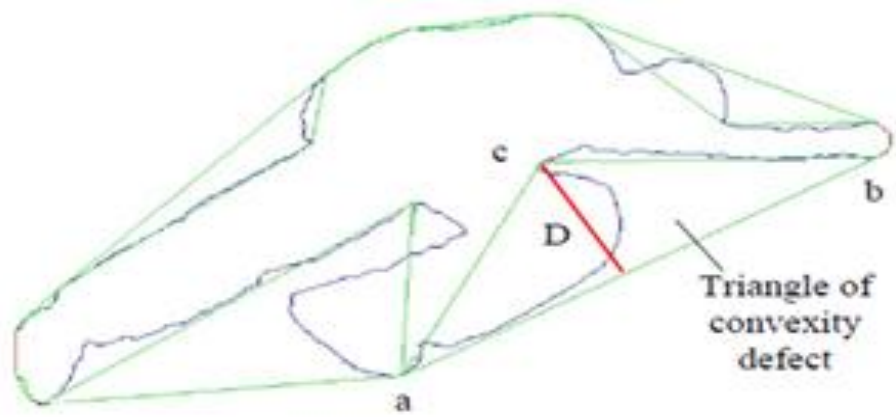

Fig. 15. Convexity defects of a contour.

Since similar contours may have different numbers of convexity defects depending on the size of the shape and the image resolution, it is more useful to calculate the distribution (histogram) of the size of the defects. In order to eliminate the dependency form the size of the shape, it is suggested in this research that for each defect the ratio of the depth of the defect and the length of the bounding box around the contour is calculated. Fig. 16 shows the smallest possible bounding box around the contour. Finally Fig. 17 depicts the 
convexity defect histogram of the contour from Fig. 15. The full range of ratios is divided into five sub-ranges.

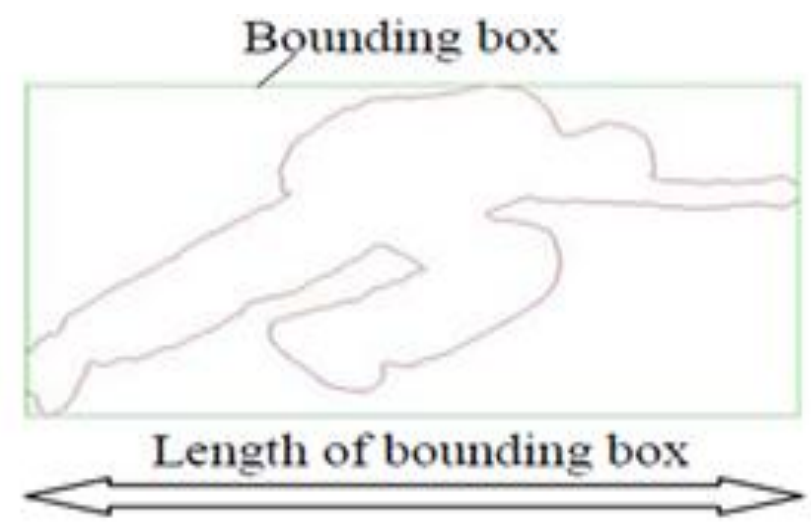

Fig. 16. Bounding box around a contour.

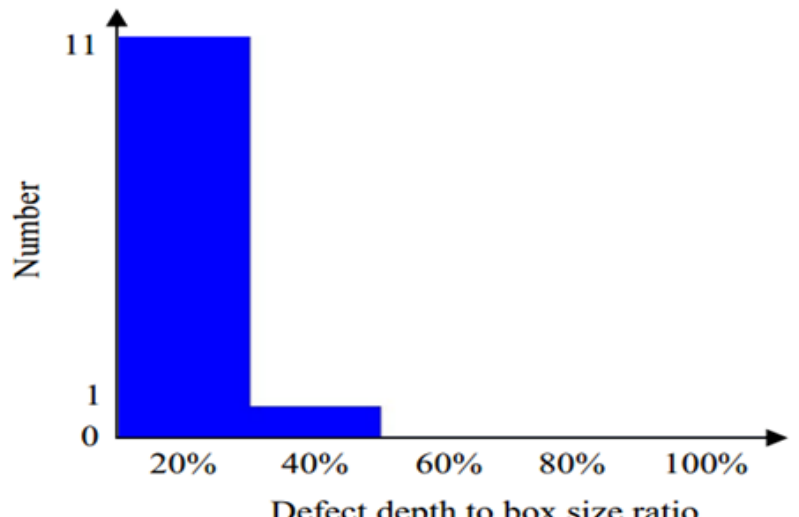

Fig. 17. Convexity defects histogram of a contour.

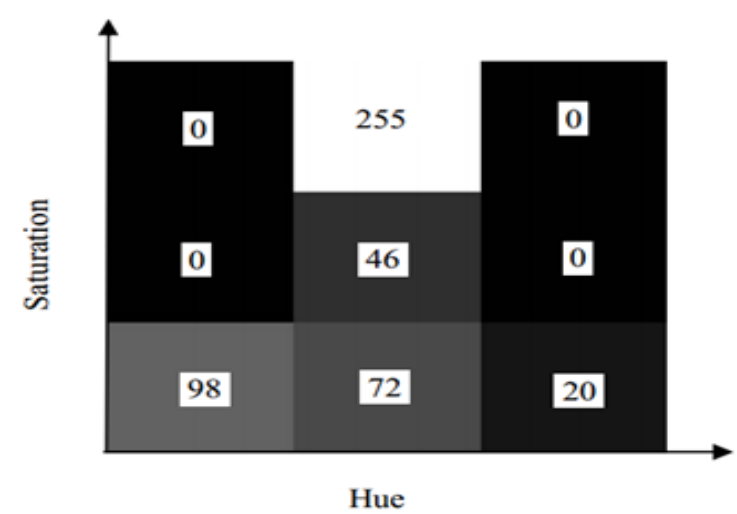

Fig. 18. Hue-saturation histogram of the image from Fig. 9.

In order to facilitate the image recognition algorithm, one more image characteristic, the image hue-saturation (HS) distribution (histogram) was added. This proved especially useful for cases where the patients were wearing uniforms. Fig. 18 shows the HS histogram of the image from Fig. 9 (combined clothing and face colours). Both parameters are divided into three sub-ranges, and the values are scaled between 0 and 255 .

To identify a patient lying on the floor based on so many parameters of the contours, a three-layer (input, hidden, output) artificial neural network (ANN) was trained using the back-propagation supervised training algorithm. Table 2 shows the number of input neurons allocated to each parameter. Values from the image 
characteristics served as inputs to the ANN. The total number of input neurons is 23 , and the same number selected experimentally for the number of neurons in the hidden layer. The output layer only contains one neuron; it shows if according to the ANN the image parameters presented at the input neurons characterise an image of a patient lying on the floor.

Table 2. Neural Network Training Parameters

\begin{tabular}{|c|c|}
\hline $\begin{array}{l}\text { No of } \\
\text { neurons }\end{array}$ & Parameter \\
\hline (FCC1-FCC8) & $\begin{array}{l}\text { Freeman chain-code histogram. } \\
\text { One neuron for each of the movement codes (see Fig.10). }\end{array}$ \\
\hline (WDCC1-DCC3) & $\begin{array}{l}\text { Weighted differential chain-code histogram. } \\
\text { One neuron for each one of the L, R, S codes (see Table II). }\end{array}$ \\
\hline$(\mathrm{CD} 1-\mathrm{CD} 3)$ & $\begin{array}{l}\text { Convexity defects histogram. } \\
\text { One neuron for each of the three sub-ranges (see Fig.17). }\end{array}$ \\
\hline (HS1-HS9) & Hue-saturation histogram. One neuron for each element of the $3 * 3$ matrix (see Fig. 18) \\
\hline
\end{tabular}

13) The ANN was presented by positive images (shapes of patients lying on the floor) and negative images (shapes that do not belong to a patient on the floor: other persons and non-human objects. The training samples were extracted from camera images; their characteristics were automatically calculated, and then presented to the input neurons of the ANN. For each training sample the solution (patient on the floor or else) was also presented to the output of the ANN.

14) If a patient is found lying on the floor, the input image is locally saved and an event 'Patient lying on the Floor' is raised through the Orca interface to be shared with the other swarm components. The event message includes the filename and location ID of the event. Then the module returns to standby mode.

\subsection{Patient Physical Condition Monitoring}

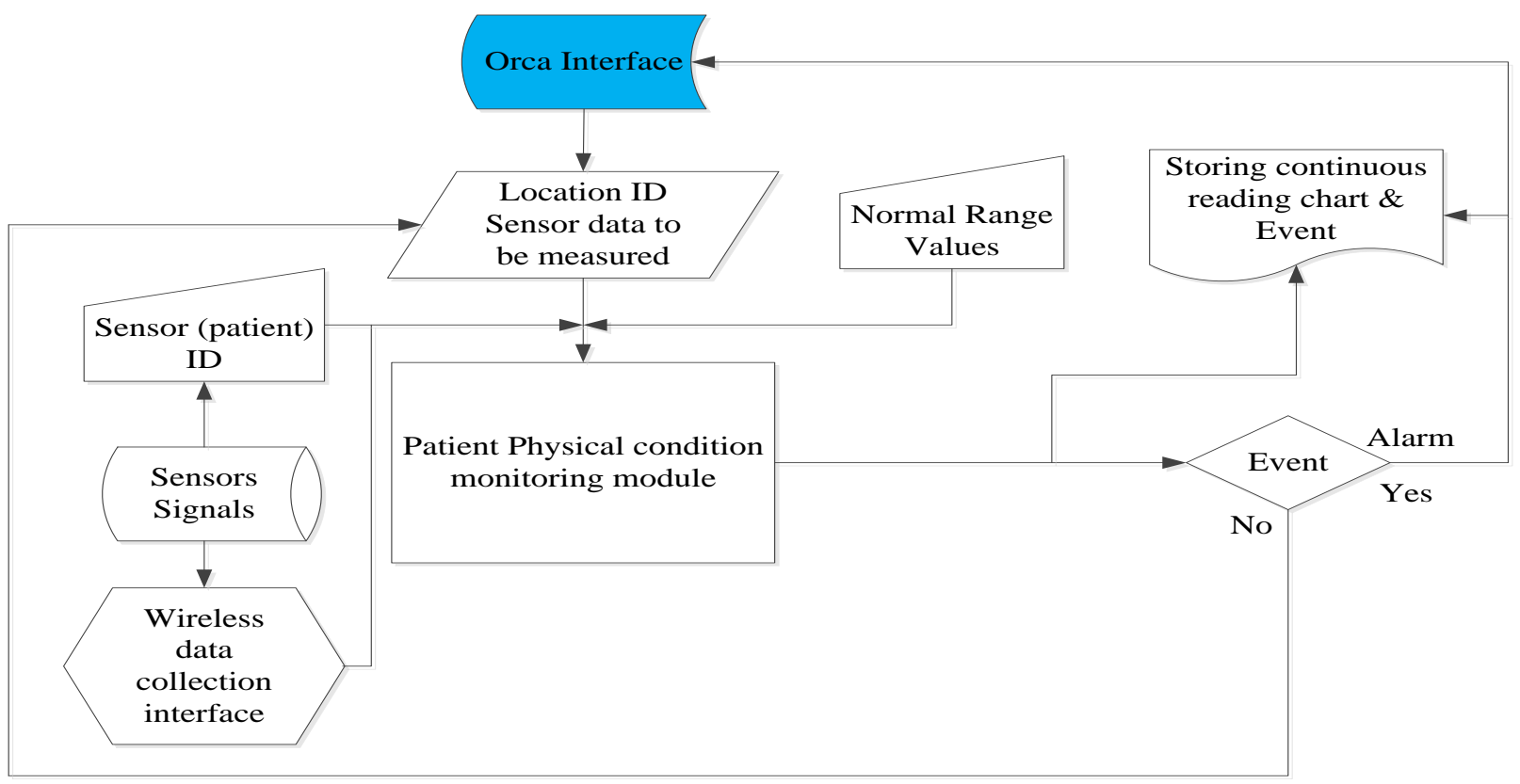

Fig. 19. Flow chart of the patient condition monitoring module.

This module is responsible to monitor patient psychical condition (i.e. vital signs: BTemp, ECG, HR, RR and BA) remotely using the Equivital [24] sensor belt. The patient physical condition monitoring software receives module activation commands through the Orca interfaces. To get the location ID, the module software also uses the Orca interface. Then the measured sensor data is compared with pre-defined values for the normal physical condition and are stored for future reference. In case of any event (i.e. high/low 
body temperature, heart rate and respiration rate), the module raises an event alarm which is shared with other swarm components by publishing it through the Orca interface. Fig. 19 shows a flow diagram of this module's software algorithm. The Patient condition monitoring software steps are as follows:

1) Gets location/patient ID where measurements to be taken.

2) Reads normal and previous physical condition values for this ID/patient from the data file.

3) Reads Bluetooth/serial port channel 'patient data', converts it according to the technical specification.

4) Calculates body temperature, ECG curve, heart rate, respiration and so on.

5) Compares the calculated results using the Identity Score (section V covers more detail) and raises event if measurements are outside range.

6) Compares current and previous measurement (if any), raises event of notification if measurements are increased or decreased.

7) If at any stage the equipment stops working, then the status would be Module_Active = NOT_WORKING and an event is raised. All operation is stopped and only 'shutdown' or 'reset' commands are accepted from other components.

\subsection{Module Communication}

The multitude of software components in the iWARD system requires a robust communication method between the components. Each software module is developed as an Orca2 component. Communication between the components is performed using specified interfaces that provide access to the component data for other (external) components. The situation recognition module uses the interfaces of the navigation and hardware manager components, and provides access to the recognised images to any external component that requires them. Fig. 20 represents a schematic diagram of the remote patient physical condition monitoring system.

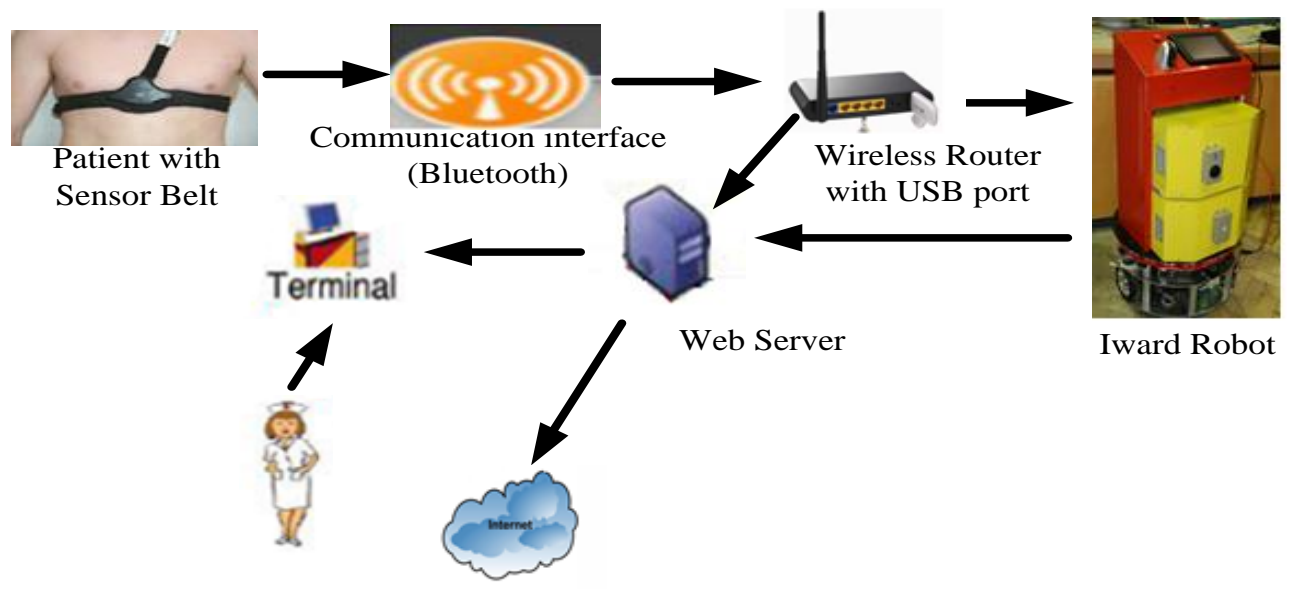

Fig. 20. Patient condition monitoring system.

\section{Condition Monitoring with Identity Detection}

The post iWARD system improved upon the prototype by implementing the identity detection methods. This allowed for the use of more robust patient condition monitoring algorithms. This research used an approach to patient monitoring that is based on novelty detection [32], in which a multivariate, multimodal model of the distribution of vital-sign data from "normal" patients is constructed. Multivariate test data $x$ are then compared with this model to give an Identity Score (IS) $z(x)$, and an alert is generated when the IS exceeds some threshold $z(x)>\kappa$. 
The vital-sign data were acquired from the EQ-01 Sensor Unit. These multivariate data were comprised of body temperature (BTemp), heart rate (HR), electrocardiogram (ECG), respiration rate (BR), body acceleration (BA).

A training set of "normal" data $\mathrm{X} \in \mathrm{RD}$, with dimensionality $\mathrm{D}=5$, was obtained from a large number of sample patients' physiological conditions selecting different ethnicity. Each dimension $d=1 \ldots D$ of Xd was normalized using a zero-mean, unit-variance transformation, [(Xd $-\mu d)(\sigma d)-1]$, where $\mu d, \sigma d$ were the mean and standard deviation of Xd, respectively. Thus, each dimension of $\mathrm{X}$ varied over approximately similar ranges.

The training set X consisted of approximately $3.1 \times 103$ data, obtained from over 400 hours of patient recording. k-means clustering [33] was used to reduce this large dataset to $\mathrm{k}=500$ prototype vectors, $\mathrm{X}$, where the 100 prototype vectors furthest from the centroid of $\mathrm{X}^{\prime}$ were discarded in order to ensure that only "normal" prototype vectors were used for construction of a model of normality.

The Parzen window method [33] was used to estimate the probability density function $p(x)$ of the remaining $\mathrm{N}=400$ prototype vectors $x_{1}, x_{2}, \ldots, x_{N}$, showed in Eq. (1). The Parzen window method [33] was used to estimate the probability density function $p(x)$ of the remaining $\mathrm{N}=400$ prototype vectors $x_{1}, x_{2}, \ldots, x_{N}$, showed in Eq. (1).

$$
p(\boldsymbol{x})=\frac{1}{\mathrm{~N}(2 \pi)^{\frac{D}{2}} \sigma^{D}} \sum_{i=1}^{N} \exp \left(-\frac{|x-x i|^{2}}{2 \sigma^{2}}\right)
$$

which is a weighted sum of Gaussian kernels centred at the 400 prototype vectors, $x_{i}$, and where each kernel has variance $\sigma 2$. This variance was estimated using the nearest-neighbor method proposed in [34]. Identity score (IS) $z(x)$ was defined as Eq. (2),

$$
z(x)=-\ln p(x)
$$

such that "normal" data, which have higher probability density values $p(x)$, take low IS, and "abnormal" data, which have lower probability density values, take high IS. An example of this: the distribution $p(x)$ for $\mathrm{D}=2$ dimensions, $\{\mathrm{HR}, \mathrm{BR}\}$.

An Identity threshold $z(x)=\kappa$ was set such that test data $\mathrm{x}$ were classified "abnormal" if $z(x)>\kappa$, and an alert was raised when $z(x)>\kappa$ for at least 2 minutes in any 4-minute window of data. The value of $\kappa$ was selected based on $[35,36]$ to minimize the false-alerts. For example, an observation of HR $=120, B R=$ 10 to be classified "abnormal" with respect to the model of normality, because the point $(120,10)$ lies outside the Identity threshold.

\section{Test, Results and Analysis}

The test of the robots during the review meetings were mainly concerned with the technical aspects of the developments: hardware and software functionality. In order to understand the usability of the system by healthcare professionals, two additional evaluation tests were organised in a teaching hospital in Newcastle (October 2009), UK and in the Matia hospital in San Sebastian (November 2009), Spain. Some thirty healthcare professionals (nurses, nursing lecturers and healthcare assistances) attended the two events. Each participant evaluated each scenario of the robot system. The general consensus of the evaluation was that a robot system consisting of small, self-navigating robots with interchangeable service modules is a good concept for making the work of healthcare professionals more efficient. Fig. 21 shows the testing of the iWARD robots during the first and second Evaluation meetings. 

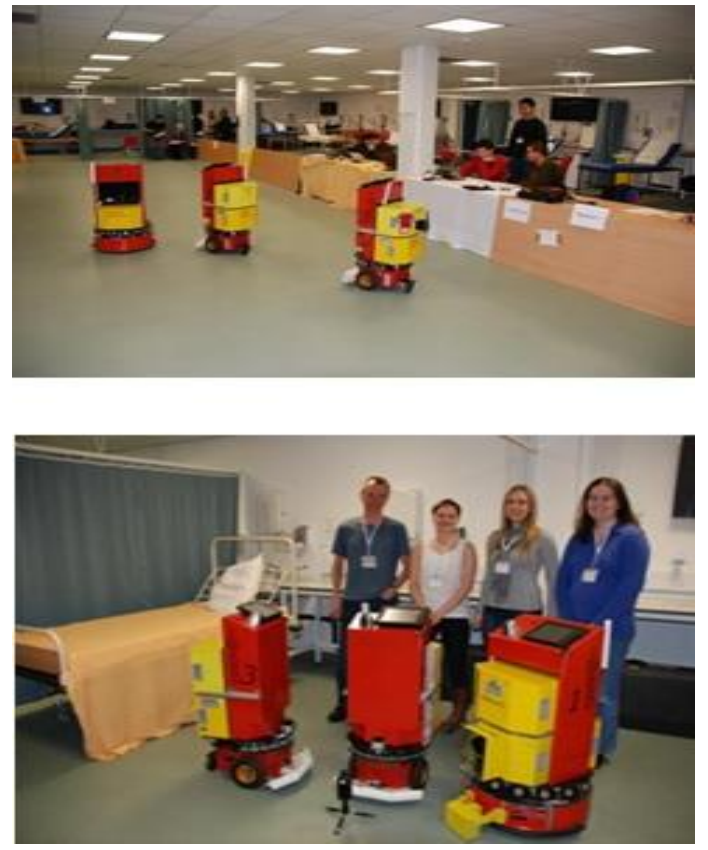

Fig. 21. Testing of the iWARD robots during the first and second Evaluation meetings (October 2009, Newcastle, and November 2009, San Sebastian).

\subsection{Recognising Patient Lying on the Floor}

The initial iWARD situation recognition module was tested during the final review meeting of the consortium and in two teaching hospitals (in Newcastle and San Sebastian). Although the test results were conclusive enough to justify the aim of the project, the lack of a laser sensor and the usage of a low-cost IP camera made the prototype module less robust. This resulted in frequent recognition errors, especially when neither the face of the person nor their badge was visible.

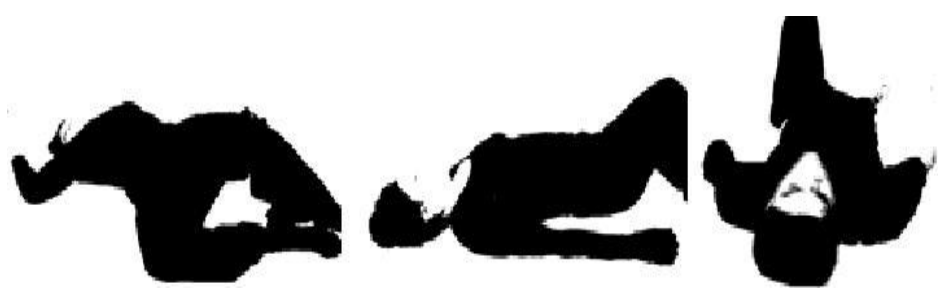

Fig. 22. Positive image samples for ANN training.
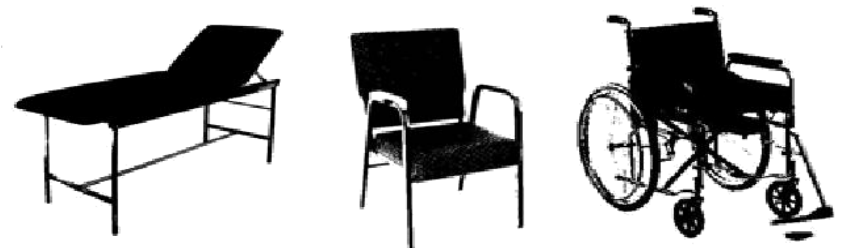

Fig. 23. Negative image samples for ANN training.

The post iWARD system improved upon the prototype by adding a 3D laser sensor and replacing the original camera with a high quality PTZ camera. This allowed for the use of more robust image recognition algorithms. The final classification decision is made by the ANN. Data containing the FCC, DFCC, convexity defects and hue-saturation properties of the object (i.e. Human and non-human classes) are the input to the multi-layered network. The ANN was presented by 6700 combination of positive images (shapes of patients 
lying on the floor, Fig. 22) and negative images (shapes that do not belong to a patient on the floor: other persons and non-human objects, Fig. 23) samples. The training samples were extracted from camera images; their characteristics were automatically calculated, and then presented to the input neurons of the ANN. For each training sample the solution (patient on the floor or else) was also presented to the output of the ANN.

After the ANN was trained a new set of about 4000 images (that did not take part in the training) were presented to the network and the solutions were calculated to test the system. During an extended test run of the system, the badge detection success rate was 95.5\% (unsuccessful recognition means that a visible badge was not identified or a random object was mistakenly identified as a badge). The contour analysis success rate for persons wearing blue hospital uniform was 93\%, whereas with persons wearing arbitrary clothing it was $81 \%$.

\subsection{Patient Physical Condition Monitoring}

The Equivital sensor provides the patient monitoring data (Fig. 24), which have been sampled in each data packet at a certain interval. For each patient monitored a unique identity number is used to distinguish the information between them. The identity number is automatically assigned to a particular patient when the communication is established. BTemp reading gives the surface body temperature (measured on the chest) for the patient. ECG Waveform, HR, BTemp, RR, BA are updated every 15 seconds and the data is recorded into excel files in webpages according to patient ID that can be read by healthcare professions remotely for viewing later for making decisions.

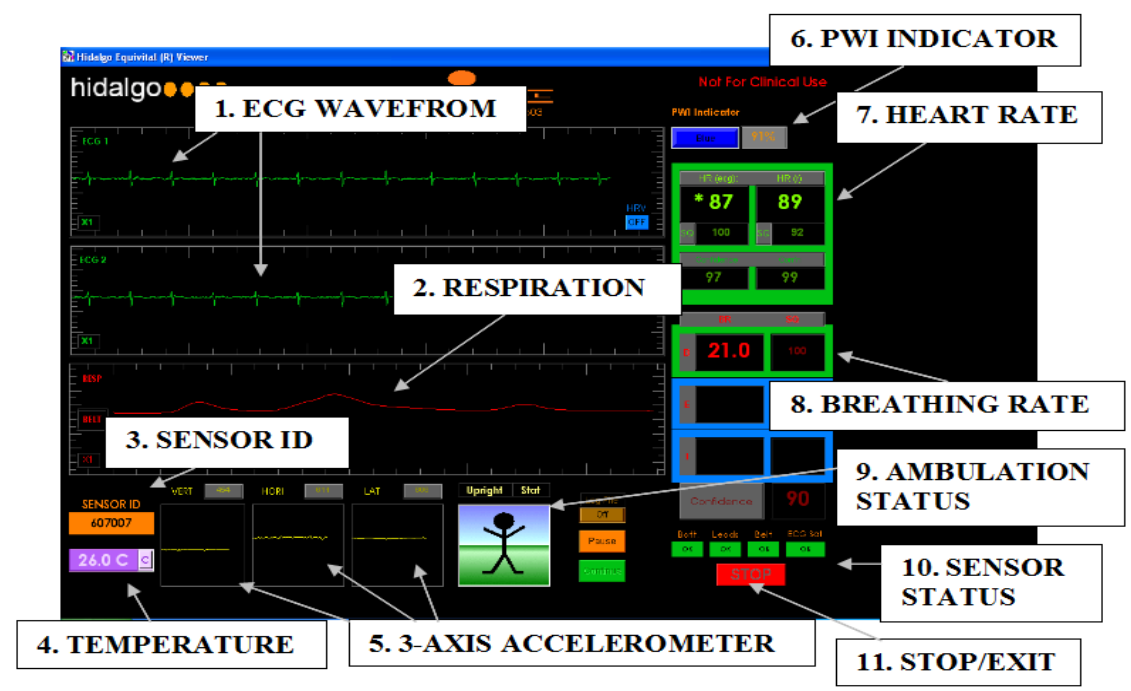

Fig. 24. Sensor electronic module Viewer.

\section{Conclusion}

The iWARD project and this research proved the concept of using a large number of small, self-navigating robots equipped with interchangeable service modules in hospitals. From the results of this research, the following major conclusions can be drawn:

- This service module can be attached to any of the hospital robots that were developed in the iWARD project. The developed modules are fully interchangeable; they have standard mechanical, power and communication connections with the mobile robot platforms.

- Communication between the module software components with other components of the system (like the hardware manager and the mission controller, both running on the main computers of the robots) is done using pre-defined interfaces of the Orca middleware software. This module can be controlled externally and 
can operate in powersave mode when inactive.

- The patient condition monitoring system remotely measures the following patient physical conditions: body temperature (STemp), heart rate (HR), electrocardiogram (ECG), respiration rate (RR), body acceleration (BA) using sensors attached to the patient's body and able to raise an alarm in the case of any abnormality detected. The module can be used as part of general monitoring or it can gather data from specific patients. The situation recognition module uses non-intrusive machine vision-based concepts, which can monitor the environment in order to detect an intruder, or a patient lying on the floor. The module can correctly identify persons (potential intruders and/or patients lying on the floor) in the majority of cases. These service modules for patient condition monitoring and situation recognition can effectively contribute to improving hospital healthcare. They can also improve the working conditions of hospital staff and reduce staff shortages.

- The overall system is effective in terms of price and performance. Manufacturing cost would be low compared to the purchase cost of specialised service robots.

\section{Acknowledgment}

The authors would like to acknowledge technicians of the Mechanical and manufacturing Engineering department (Dublin City University) for their assistance during this project.

\section{References}

[1] Intelligent robot swarm for attendance. Recognition, Cleaning and Delivery. Retrieved, 2014, from: www.iward.eu

[2] Orca robotics. Retrieved 2014, from http://orca-robotics.sourceforge.net

[3] Open source computer vision library (OpenCV). Retrieved 2014, from: http://opencv.org

[4] Griffin, C., \& Szecsi, T. (2008). Development of an automated cleaning system for hospitals. Proceedings of the 4-th Virtual International Conference on Intelligent Production Machines and Systems (IPROMS). Cardiff, UK, Elsevier Ltd., Oxford.

[5] European information society and TNO innovation for life, robotics for healthcare. Retrieved 2014, from:

http://ec.europa.eu/information_society/activities/health/docs/studies/robotics_healthcare/robotics -in-healthcare.pdf

[6] Skilligent, Healthcare/Eldercare Robot based on Skilligent Technology. Retrieved 2014, from: http://www.skilligent.com/products/documents/docs/Skilligent-Health-Elder-Care-Robot-Study.pdf

[7] Cao, Y Y. U., Fukunaga, A. S., \& Khang, A. B. (1997). Cooperative mobile robotics: Antecedents and directions. Autonomous Robots, 4(1), 7-27.

[8] Brooks, R. A. (1986). A robust layered control system for a mobile robot. IEEE Journal of Robotics and Automation, 2(1), pp. 14-23.

[9] Matari'c, M. J. (1995). Issues and approaches in the design of collective autonomous agents. Robotics and Autonomous Systems, 16, 321-331.

[10] Rosenblatt, J. K. (1997). DAMN: A distributed architecture for mobile navigation. Journal of Experimental Theoretical Artificial Intelligence, 339-360.

[11] Parker, L. E. (1997). L-ALLIANCE: Task-oriented multi-robot learning in behavioral-based systems. Advanced Robotics, 11(4), 305-322.

[12] Donald, B. R. (1995). Information invariants in robotics. Artificial Intelligence, 72(1-2), 217-304.

[13] Parker, L. E. (1998). Toward the automated synthesis of cooperative mobile robot teams. Proceedings of 
the SPIE Mobile Robots XIII (pp. 82-93).

[14] Ambrose, R., \& Askew. R. S. (1995). An experimental investigation of actuators for space robots. Proceedings of IEEE International Conference on Robotics and Automation (pp. 2625 - 2630).

[15] Hayes, A. T., Martinoli, A., \& Goodman, R. M. (2002). Distributed odour source Localization. IEEE Sensors Journal, 2(3).

[16] Monkman, G. J., \& Taylor, R. D. (1993). Thermal tactile sensing. IEEE Transactions on Robotics and Automation, 9.

[17] Shieh, M. Y., Hsieh, J. C., \& Cheng, C. P. (2004). Design of an intelligent hospital service robot and its applications. IEEE International Conference on Systems, Man and Cybernetics, 4377-4382.

[18] Takahashi, M., Suzuki, T., Cinquegrani, F., Sorbello, R., \& Pagello, E. (2009). A mobile robot for transport applications in hospital domain with safe human detection algorithm. Proceedings of the IEEE International Conference on Robotics and Biomimetics (ROBIO) (pp. 1543-1548).

[19] Rafflin, C., \& Fournier, A. (1995). Learning with a friendly interactive robot for service tasks in hospital environments. Proceedings of the IEEE/RSJ International Conference on Human Robot Interaction and Cooperative Robots Proceedings (pp. 492-497).

[20] University of massachusetts amherst. Retrieved 2014, from: www-robotics.cs.umass.edu/index.php/Robots/UBot-5

[21] University of Massachusetts Amherst, Robots assist with elder care. Retrieved 2014, from: www.cs.umass.edu/csinfo/announce/robots_elder.html

[22] Abus TV 240 IP camera. Retrieved 2014, from: www.appzer.de/ipcamera/ABUS_TV7240.html

[23] Ifm electronics, PMD 3D. Retrieved 2014, from: http://www.ifm.com/ifmirl/web/pmd.htm

[24] Equivital. [Retrieved 2014, from: http://www.equivital.co.uk

[25] Liu, Y.-K., Zalik, B., Wang, P.-J., \& Podgorelec, D. (2012). Directional difference chain codes with quasi-lossless compression and run-length encoding. Proceedings of the Image Communication (pp. 973-984).

[26] Liu, Y.-K., Wei, W., Wang, P.-J., \& Zalik, B. (2007). Compressed vertex chain codes. Pattern Recognition, 40 (11), 2908-2913.

[27] Sanchez-Cruz, H. (2010). Proposing a new code by considering pieces of discrete straight lines in contour shapes. J. Vis. Commun. Image Ricognition, 21(4), 311-324.

[28] Borut, Ž., \& Niko, L. (2014). Chain code lossless compression using move-to-front transform and adaptive run-length encoding. Signal Processing: Image Communication, 29(1), 96-106.

[29] Andrew, J. N., \& Lewis, D. G. (2014). Writer identification using oriented basic image features and the delta encoding. Pattern Recognition, 47(6), 2255-2265.

[30] Freeman, H. (1961). On the encoding of arbitrary geometric configurations. IEEE T. Electron, 10(2), 260-268.

[31] OpenCV. Retrieved August 2014, from: http://opencv.willowgarage.com

[32] Hann, A. (2008). Multi-parameter monitoring for early warning of patient deterioration. Ph.D. thesis, University of Oxford.

[33] Richard, O. D., Peter, E. H., \& David, G. S. (2010). Pattern Classification. John Wiley and Sons, New York, 2 edition.

[34] Bishop, C. M. (1994). Novelty detection and neural network validation. Proceedings of IEE Conference on Vision and Image Signal Processing, 141(4), 217-222.

[35] Pulse and respiration. [Videocassette], Vital signs Series 1, School of Nursing, Dublin City University, Ireland.

[36] Barbara, K. (1998). Assessing vital signs. Fundamentals of Nursing. (5th ed.), Addison -Wesley, Canada, 
pp.425-448.

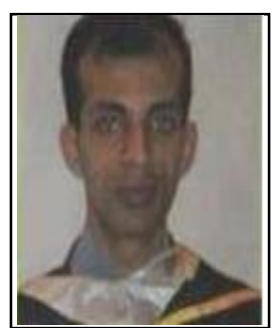

Kabir Mamun received his bachelor of engineering (2003) degree from Islamic University of Technology, Bangladesh. He received his master of engineering (By Research, 2007) degree and the Ph.D. (2012) degree from Dublin City University (DCU), Ireland, in mechanical and manufacturing engineering.

He joined the University of the South Pacific (USP) in 2013 as a lecturer. He also worked as a mechanical and instrumentation design engineer at Sonex Metrology Ltd. which is a metrology tools design company for the semiconductor industries. He has work experience as a research assistant at the School of Mechanical and Manufacturing Engineering in the prestigious Dublin City University (DCU). He started his professional career as an assistant production/manufacturing engineer in Sun power Ceramic industry. He reviewed several articles of several journals.

Dr. Kabir is a member of the Institute of Electrical and Electronics Engineers (IEEE) and treasurer of the IEEE (R10) Fiji Sub-section.

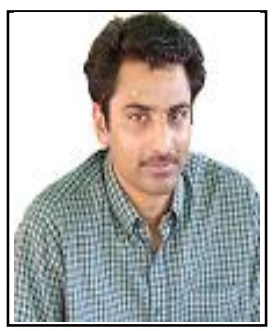

Alok Sharma received the B.Tech. degree from the University of the South Pacific (USP), Suva, Fiji, in 2000 and the M.Eng. degree, with an academic excellence award, and the Ph.D. degree in the area of pattern recognition from Griffith University, Brisbane, Australia, in 2001 and 2006, respectively.

He was with the University of Tokyo, Japan (2010-2012) as a research fellow. He is an a/prof. at the USP and an adjunct a/prof. at the Institute for Integrated and Intelligent Systems (IIIS), Griffith University. He participated in various projects carried out in conjunction with Motorola (Sydney), Auslog Pty., Ltd. (Brisbane), CRC Micro Technology (Brisbane), the French Embassy (Suva), and JSPS (Japan). His research interests include pattern recognition, computer security, human cancer classification and protein fold and structural class prediction problems. He reviewed several articles and is in the editorial board of several journals.

Dr. Alok is a member of the Institute of Electrical and Electronics Engineers (IEEE).

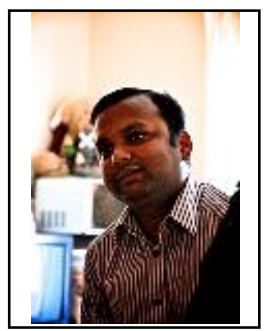

A. S. M. Hoque received his bachelor of engineering (2002) degree from Islamic University of Technology, Bangladesh. He received his Ph.D. (2010) degree from Dublin City University (DCU), Ireland, in mechanical and manufacturing engineering.

$\mathrm{He}$ is an assistant professor in the Department of Industrial and Production Engineering (IPE) at Jessore University of Science and Technology (JUST), Bangladesh since 2010. He reviewed several articles of several journals.

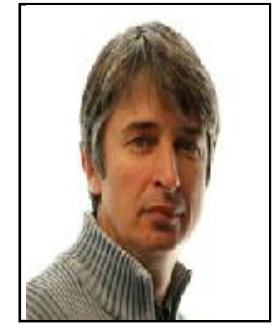

Tamas Szecsi graduated with a degree in manufacturing automation from the Technical University 'MOSSTANKIN', Moscow, Russia, in 1983. He worked one year at the tool making factory 'SZIM', Kecskemet, Hungary, as a designer. He worked at the University of Rousse, Bulgaria, as assistant lecturer, lecturer, senior lecturer and associate professor (January 1985- June 1999). Research in the area of Cutting tool condition monitoring lead him to a Ph.D. degree in Manufacturing Engineering which he received from the University of Rousse in 1997.

He took up a lecturer position (1999) in manufacturing processes and systems at the School of Mechanical and Manufacturing Engineering at DCU. Since 2002 he is a senior lecturer at the same School. He reviewed several articles and is in the editorial board of several journals. 